\section{Determination of Transverse Wave Velocities in Solids}

A TECHNIQUE for finding longitudinal wave velocities in solids has already been described $^{1}$; velocities of transverse waves can be determined with the same apparatus.

Ultrasonic waves were generated by an oscillator connected to a quartz crystal. The waves were received by another similarly cut quartz crystal. The received waves were detected and measured by a two-stage radio-frequency amplification, followed by single stages of rectification and D.c. amplification. Screening of the detector set had to be thorough, so that it would not pick up any general electromagnetic radiation from the oscillator set. In this detail, the method for transverse waves differs from the method for longitudinal waves, where sufficient leakage of electromagnetic waves into the detector had purposely to be provided ${ }^{1}$.

The crystals were placed flat upon solid surfaces, such as of concrete, marble, iron, copper, ebonite. Thin layers of glycerine were smeared on the surfaces for maintaining 'acoustic' continuity between quartz and the solid surfaces. A frequency of $300 \mathrm{Kc} / \mathrm{sec}$. was used. As the oscillator erystal was moved away or towards the detector crystal (of course, either can be moved, whichever is practicable), the intensity of pick-up of ultrasonics was found to pass through maxima and minima. The distances between consecutive shifts for two maxima or two minima must be the half wave-length of the sound waves. Unfortunately, the programme of research had to be broken off at this stage, and no accurate measurements of the wave-length could be made; but the approximate measurements supported the theoretical expectation that the distances measured were half wave-lengths.

This technique has the advantages over the 'lycopodium powder' method of actually marking out the sound field by differential distribution of the powder by the sound on the solid surface, that only a very weak source of ultrasonics (fraction of a watt) is necessary, accurate measurements can be made of the shift of the movable crystal, the application of the method to field measurements for extended, immovable solids, etc.

Physical Laboratories,

\section{R. Parshad}

Council of Scientific and Industrial Research, University Buildings, Delhi.

Sept. 18.

${ }^{1}$ Parshad, R., Nature, 156, 637 (1945).

\section{Friedel-Crafts Polymerizations}

$\alpha$-METhYLSTYRENe has been polymerized at $25^{\circ} \mathrm{C}$, using stannic chloride as catalyst, both in solution and in undiluted monomer, giving polymers of molecular weight up to approximately 11,000. Staudinger ${ }^{1}$, using this catalyst, obtained only a very low degree of polymerization (up to octamer), presumably due to the high temperatures reached in his experiments. The molecular weight of the polymer can be increased three-fold by polymerizing at $0^{\circ} \mathrm{C}$., in agreement with the aluminium chloride-catalysed reaction ${ }^{2}$.

The opinion is widely held ${ }^{3,4,5}$ that polymerizations of this type (catalysed by acids, boron trifluoride, aluminitum chloride, stannic chloride, etc.) proceed

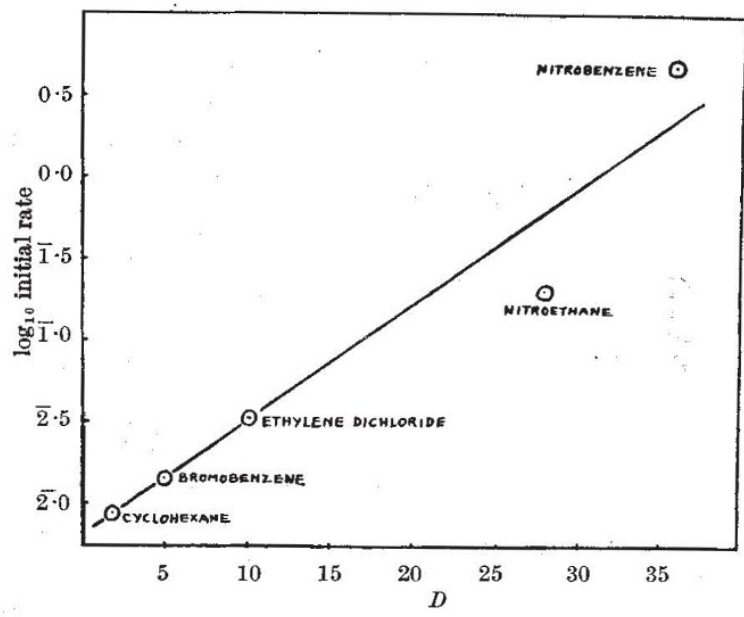

by an ionic chain process. Direct evidence of such a mechanism is provided by the observation that both the rate and degree of polymerization of $\alpha$. methylstyrene are increased by increase in the dielectric constant of the solvent. Similar effects are well established in simple ionie reactions ${ }^{6, ?}$.

The accompanying graph shows a plot of $\log _{10}$ (initial rate) at $25^{\circ} \mathrm{C}$. against dielectric constant (of the solvent) over a range from $D=1.9$ (cyclohexane) to $D=36$ (nitrobenzene), for an initial concentration of monomer of 1.36 moles/litre and catalyst of $9 \times 10^{-4}$ moles/litre. (Rate $=$ disappearance of monomer in moles/litre/min. ${ }^{-1}$.) Over this range the molecular weight (number average) increases in a similar marked fashion, as shown in the table below.

$\begin{array}{lcc}\quad \text { Solvent } & \text { Dielectric constant } & \text { Molecular weight } \\ \text { Cyclohexane } & 1 \cdot 9 & 500 \\ \text { Ethylene dichloride } & 10 & 1200 \\ \text { Nitro-etháne } & 28 & (680) \\ \text { Nitrobenzene } & 36 & 8500\end{array}$

There is, however, some quantitative uncertainty here, as it is difficult to remove traces of monomer from the polymer.

Until the effects of monomer and catalyst concentration have been fully determined, it will not be possible to deduce the formal kinetic steps. But if it may be assumed that these are the same in all the above solvents, and also that a stationary state is established, conclusions can be drawn about their nature. For a given monomer and catalyst concentration, the rate and degree of polymerization will depend upon the specific rate constants for initiation $\left(k_{i}\right)$, propagation $\left(k_{p}\right)$, and termination $\left(k_{t}\right)$ as follows :

$$
\text { Rate } \propto \frac{k_{i} \cdot k_{p}}{k_{t}} ;
$$

Degree of polymerization (number average) $\propto \frac{k_{p}}{k_{t}}$.

The dielectric constant may, in principle, influence any or all of these rate constants, since all are likely to involve ionic processes. But the fact that both the rate and the degree of polymerization are similarly affected indicates that its main influence is on the ratio $k_{p} / k_{t}$ rather than on $k_{i}$.

If the propagation step is the reaction of a monomer molecule with an ionic active centre, as generally assumed ${ }^{4,5}$, then by analogy with simple reactions between neutral molecules and ions ${ }^{7}$ we should expect $k_{p}$ to be reduced by increase in dielectric constant. An increase in the ratio $k_{p} / k_{t}$ can, therefore, only be secured by a similar but greater decrease in $k_{t}$. This 